\title{
Association between aneurysm hemodynamics and wall enhancement on 3D vessel wall MRI
}

\author{
Muhammad Owais Khan, PhD, ${ }^{1,2}$ Veronica Toro Arana, BS, ${ }^{3}$ Christian Rubbert, MD, ${ }^{4}$ \\ Jan F. Cornelius, MD, ${ }^{5}$ Igor Fischer, PhD, ${ }^{6}$ Richard Bostelmann, MD, ${ }^{5}$ \\ Hendrik-Jan Mijderwijk, MD, MSc, PhD, ${ }^{5}$ Bernd Turowski, MD, ${ }^{4}$ Hans-Jakob Steiger, MD, ${ }^{5}$ \\ Rebecca May, MD, ${ }^{4}$ and Athanasios K. Petridis, MD ${ }^{5}$
}

\begin{abstract}
1'Department of Pediatrics, ${ }^{2}$ Institute for Computational and Mathematical Engineering, and ${ }^{3}$ School of Medicine, Stanford University, Stanford, California; ${ }^{4}$ Medical Faculty, Department of Diagnostic and Interventional Radiology, University Düsseldorf, Germany; and ${ }^{5}$ Department of Neurosurgery and ${ }^{6}$ Division of Informatics and Data Science, Department of Neurosurgery, University Hospital Düsseldorf, Germany
\end{abstract}

OBJECTIVE Aneurysm wall enhancement (AWE) on 3D vessel wall MRI (VWMRI) has been suggested as an imaging biomarker for intracranial aneurysms (IAs) at higher risk of rupture. While computational fluid dynamics (CFD) studies have been used to investigate the association between hemodynamic forces and rupture status of IAs, the role of hemodynamic forces in unruptured IAs with AWE is poorly understood. The authors investigated the role and implications of abnormal hemodynamics related to aneurysm pathophysiology in patients with AWE in unruptured IAs.

METHODS Twenty-five patients who had undergone digital subtraction angiography (DSA) and VWMRI studies from September 2016 to September 2017 were included, resulting in 22 patients with 25 IAs, 9 with and 16 without AWE. High-resolution CFD models of hemodynamics were created from DSA images. Univariate and multivariate analyses were performed to investigate the association between AWE and conventional morphological and hemodynamic parameters. Normalized MRI signal intensity was quantified and quantitatively associated with wall shear stresses (WSSs) for the entire aneurysm sac, and in regions of low, intermediate, and high WSS.

RESULTS The AWE group had lower WSS $(p<0.01)$ and sac-averaged velocity $(p<0.01)$ and larger aneurysm size $(p<0.001)$ and size ratio $(p=0.0251)$ than the non-AWE group. From multivariate analysis of both hemodynamic and morphological factors, only low WSS was found to be independently associated with AWE. Sac-averaged normalized MRI signal intensity correlated with WSS and was significantly different in regions of low WSS compared to regions of intermediate $(p=0.018)$ and high $(p<0.001)$ WSS.

CONCLUSIONS The presence of AWE was associated with morphological and hemodynamic factors related to rupture risk. Low WSS was found to be an independent predictor of AWE. Our findings support the hypothesis that low WSS in IAs with AWE may indicate a growth and remodeling process that may predispose such aneurysms to rupture; however, a causality between the two cannot be established.

https://thejns.org/doi/abs/10.3171/2019.10.JNS191251

KEYWORDS hemodynamics; computational fluid dynamics; aneurysms; vessel wall MRI; vascular disorders

I NTRACRANIAL aneurysms (IAs) occur in $4 \%$ of the general population but are usually asymptomatic unless rupture occurs. ${ }^{38}$ For unruptured IAs, the risks associated with treatment and subsequent outcomes can often exceed the natural risk of rupture, and thus determining patient-specific criteria for predicting instability is critical for therapeutic decision-making. Recently, high-resolution vessel wall MRI (VWMRI) enhancement has been used to assess IA wall pathology as a surrogate marker of wall inflammation, which is an important risk factor for rup-

ABBREVIATIONS AComA = anterior communicating artery; $\mathrm{AIC}=$ Akaike information criterion; $\mathrm{AR}=$ aspect ratio (ratio of aneurysm dome height to neck width); $\mathrm{AUC}=$ area under the ROC; AWE = aneurysm wall enhancement; BNF = bottleneck factor (ratio of aneurysm dome to neck width); CFD = computational fluid dynamics; HW = height to width parameter (ratio of aneurysm height to width); IA = intracranial aneurysm; ICA = internal carotid artery; ISUIA = International Study of Unruptured Intracranial Aneurysms; MCA = middle cerebral artery; MRI-SIF = MRI-SI factor; NSI = nonsphericity index; OSI = oscillatory shear index; $\mathrm{PC}$ ComA = posterior communicating artery; $\mathrm{ROC}=$ receiver operating characteristic; SI = signal intensity; SPI = spectral power index; SR = size ratio (ratio of aneurysm size to average diameter of the parent arteries); VWMRI = vessel wall MRI; WSS = wall shear stress; WSS $^{*}=$ WSS normalized to the parent artery WSS

SUBMITTED May 3, 2019. ACCEPTED October 25, 2019.

INCLUDE WHEN CITING Published online January 10, 2020; DOI: 10.3171/2019.10.JNS191251. 
ture.$^{24}$ Hemodynamic forces are thought to play a key role in IA wall deterioration but are difficult to reliably assess through current imaging modalities.

Over the past decade, computational fluid dynamics (CFD) has emerged as a promising tool to noninvasively evaluate the hemodynamic forces to which the IA wall is exposed, leading to IA wall deterioration, particularly wall shear stress (WSS), and thus CFD can be used to investigate the underlying biomechanical phenomena involved in aneurysm pathophysiology. ${ }^{6,39}$ Although CFD studies have retrospectively characterized hundreds of aneurysms according to their rupture status, the use of CFD is not without criticism. Skepticism has been aimed toward CFD modeling assumptions and uncertainties, ${ }^{20-22}$ sensitivity of reconstructed geometry to imaging modality, ${ }^{13,16}$ segmentation variability, 3,34 impact of rigid rather than compliant walls, ${ }^{1,9}$ and disagreement over whether low or high WSS is correlated with aneurysm rupture. ${ }^{6,39}$ Despite these controversies, CFD has recently begun to move beyond predicting association with rupture status and toward linking WSS to wall inflammation measured through histology $y^{4,24}$ and VWMRI. ${ }^{40}$

In recent years, aneurysm wall enhancement (AWE) measured through VWMRI has been shown to be a marker of rupture-prone walls. We hypothesized that abnormal hemodynamic conditions may be associated with the presence of AWE. In this study, we investigated possible correlations between AWE and CFD-derived metrics to understand the role of hemodynamic forces in aneurysm wall degradation.

\section{Methods \\ Patients}

This study was approved by the institutional review board of the University Hospital Düsseldorf (study number 6186R). From September 2016 to September 2017 (1 year), 22 patients with 25 unruptured IAs were included. All patients underwent both DSA and VWMRI within 6 weeks. IAs were considered unruptured on the basis of clinical presentation (no history of subarachnoid hemorrhage, absence of recent or past acute headaches) and imaging data.

\section{Imaging Protocol}

VWMRI was acquired on a 3T MR scanner (Magnetom Skyra, Siemens) with a 20-channel head coil. The protocol included a vendor-supplied isometric 3D T1 sampling perfection with application-optimized contrasts using different flip angle evolution (SPACE) turbo spin echo (TSE) sequences with spectral attenuated inversion recovery (SPAIR) and blood suppression (field of view $179 \times$ 230 , repetition time/echo time $939 / 18 \mathrm{msec}$, matrix $200 \times$ 256, spatial resolution $0.9 \times 0.9 \times 0.9 \mathrm{~mm}$ ) before and after administration of gadoteridol $(0.2 \mathrm{ml} / \mathrm{kg}$ body weight, maximum $20 \mathrm{ml}$; ProHance, Bracco Imaging). The "black blood" effect, i.e., the suppression of signal of the flowing blood, was achieved by the normal flow void effects of fast-flowing blood in TSE sequences, i.e., the excited molecules leaving the imaging plane before signal recording. To further minimize artifacts and even suppress the signal of slow-flowing blood, the signal of flowing blood was fur- ther attenuated using a pair of nonselective and selective inversion radiofrequency pulses. In principle, the imaging was optimized so that inflowing blood had no transverse magnetization and thus yielded no signal during readout. The scan time for the sequence was 7 minutes and $55 \mathrm{sec}-$ onds.

\section{Digital Subtraction Angiography}

Informed consent for diagnostic, clinical, and biplanar procedures was obtained from all study participants. DSA was performed and standard projections (posterior-anterior, lateral, and oblique) were obtained during manual injection of an 8- to 10-ml bolus of a contrast-enhancing agent (Accupaque $300 \mathrm{mg} \mathrm{J} / \mathrm{ml}$, GE Healthcare). 3D rotational angiograms $\left(88 \mathrm{kV}, 200 \mathrm{mAs}, 180^{\circ}\right.$, rotation time of 4.1 seconds, 122 images, manual injection of up to $20 \mathrm{ml}$ contrast-enhancing agent during the whole rotation) were obtained for the anterior circulation and on an as-needed basis for the posterior circulation.

\section{VWMRI Analysis}

The images were independently reviewed by 2 readers (R.M. with 8 and B.T. with 20 years of experience in vascular neuroimaging) who determined the presence or absence of complete or partial AWE on postgadolinium VWMRI. Multiplanar oblique reconstructions were obtained from precontrast- and postcontrast-enhanced 3D VWMRI and analyzed after coregistration. In case of disagreement among the 2 readers, a consensus was reached through a joint review and discussion of the cases.

\section{Morphological Parameters}

Morphological parameters were defined and computed as previously reported. ${ }^{10,12}$ Briefly, the size of the aneurysm was defined as the maximum diameter of the aneurysm dome, and aspect ratio (AR) was defined as the ratio of dome height to neck width, where height is the longest dimension from the neck to dome tip and width is measured perpendicular to dome height. Size ratio (SR) was measured as the ratio of aneurysm size to average diameter of the parent arteries. The bottleneck factor (BNF) was defined as the ratio of dome to neck width, and the height to width parameter (HW) was defined as the ratio of aneurysm height to width. ${ }^{12}$ To qualitatively measure the aneurysm shape, a nonsphericity index (NSI) was computed as defined previously. ${ }^{10}$ Two observers, a neurosurgeon (A.K.P.) and a neuroradiologist (B.T.), made the analysis independently of each other, and both had more than 18 years of clinical experience. The interobserver variability was minimal, and thus further statistical analysis of this variable was not performed.

\section{CFD Modeling}

DSA images were segmented and reconstructed into a 3D model using the open-source Vascular Modeling Toolkit (http://www.vmtk.org). Variable density volumetric meshes were generated with the highest mesh density in the aneurysm sac, where the tetrahedron side length was $0.12 \mathrm{~mm}$ on average, previously shown through a 320 -fold mesh-refinement study to accurately model IA hemody- 
namics. ${ }^{22}$ The number of tetrahedral elements was 4.1 million on average, ranging from 1.3 to 8.7 million, reflecting the variability in IA sizes and extent of CFD model domains.

A representative older adult internal carotid artery (ICA) flow waveform shape ${ }^{14}$ was scaled to the mean cycle-averaged cross-sectional velocity of $0.27 \mathrm{~m} / \mathrm{sec}$, which was applied to the ICA inlet. The resulting mean ICA flow rate was $4.7 \mathrm{ml} / \mathrm{sec}$, which is comparable to the in vivo measured flow rate of $4.3 \pm 0.8 \mathrm{ml} / \mathrm{sec}^{41}$ For middle cerebral artery (MCA) and anterior communicating artery (AComA) aneurysms, the inlet flow waveform was dampened by $10 \%$ to account for the transit from cervical to cavernous segments. A fully developed Womersley profile was imposed at the ICA inlet, and the flow division to the outlet segments was scaled based on the area.

CFD simulations were performed using a minimally dissipative and energy-preserving solver, ${ }^{32}$ previously verified and validated to accurately model aneurysmal flows ${ }^{2,22}$ and cardiovascular flows in general. ${ }^{18}$ We assumed rigid walls, blood viscosity of $0.0035 \mathrm{~m}^{2} / \mathrm{sec}$, and blood density of $1060 \mathrm{~kg} / \mathrm{m}^{3}$. All simulations were run for 3 cycles, with 10,000 time steps per cardiac cycle, and the last cycle was used for postprocessing. For all cases, we computed sacaveraged values for the following variables: time- and sacaveraged WSS, oscillatory shear index (OSI), and spectral power index (SPI), a recently introduced metric for quantifying high-frequency flow instabilities. ${ }^{19}$ Since WSS could have uncertainties due to CFD modeling assumptions, we normalized the WSS to the parent artery WSS (WSS*) to dampen these effects.

\section{Image Registration and Quantification of VWMRI Enhancement}

CFD models were registered to postcontrast VWMRI using an in-house landmark-based image registration tool. To quantify the AWE on postcontrast VWMRI, we projected the MRI signal intensity (MRI-SI) onto the CFD model and normalized it according to the nominal intensity of the image volume (Fig. 1). More specifically, we quantified MRI-SI along a line that was normal to each mesh node. The MRI-SI was then averaged along the length of the line and normalized to the mean MRI-SI of the entire VWMRI volume (MRI-SI factor [MRI-SIF]). As seen in Fig. 1C, the maximum MRI-SIF corresponded to the region that showed notable AWE on postcontrast VWMRI. Similar to hemodynamics parameters, we computed sac-averaged MRI-SIF as a quantitative measure of AWE. In addition, we computed MRI-SIF in low-, intermediate-, and high-aneurysm WSS regions of the sac, which were defined as regions with $<10 \%, 10 \%-30 \%$, and $>30 \%$ of parent artery WSS, respectively.

\section{Statistical Analysis}

Univariate Analysis of Differences Between the AWE and NoAWE IA Groups

Since there were only 25 observations, a Shapiro-Wilk test was performed to determine if a parameter was normally distributed. For nonnormally distributed parameters, normality was achieved by appropriate nonlinear transformation (square root or natural logarithm). In subsequent analysis, the parameter form (i.e., the raw parameter or its transformation) with the highest $\mathrm{p}$ value (the one most likely to be normally distributed) was used. The statistical significance of the observed mean differences was assessed using a 2-tailed independent Student t-test, and a $\mathrm{p}$ value $<0.05$ was considered statistically significant. Frequency ratios for nominal variables (hypertension, sex, location, smoking, hyperlipidemia, diabetes, and side of aneurysm) were compared using the Fisher's exact test.

\section{Multivariate Logistic Regression on Significant Variables}

The parameters found to be significant $(\mathrm{p}<0.05)$ in the univariate analysis were further analyzed using logistic regression to predict AWE. Potential predictor variables were forward selected based on the $p$ value from the univariate $t$ test. Variables with $p>0.1$ were discarded. Remaining variables were ranked in ascending order based on $\mathrm{p}$ value and introduced one by one into the multivariate model, starting with the one with the lowest $\mathrm{p}$ value. At each step, the Akaike information criterion (AIC) and the significance of the coefficients were checked. A predictive model relying on raw, nontransformed variables was also developed. The performance of both models for accuracy and the AUC (area under the receiver operating characteristic [ROC] curve) were assessed. Lastly, both models were internally validated and calibrated using bootstrapping with 1000 resamples.

\section{Correlation Between MRI-SIF and WSS in the AWE Group}

MRI-SIF sac-averaged means and standard deviations and MRI-SIF in low-, intermediate-, and high-WSS* regions were computed. Linear correlations and Pearson chi-square test were used to evaluate the relationship between MRI-SIF and WSS* in the AWE group.

\section{Results}

\section{Qualitative Hemodynamic Characteristics}

A total of 25 unruptured IAs were visible in 22 patients, of which 9 aneurysms showed AWE and 16 showed no AWE. Table 1 shows patient clinical characteristics and IA locations, which were not found to be associated with AWE (all $p$ values $>0.05$ ). Figure 2 shows WSS and OSI maps, along with the pre- and postenhanced images, ranked from lowest to highest WSS. These qualitative maps indicate that IAs in the AWE group had larger size and lower WSS compared with those in the no-AWE group. Moreover, IAs with the lowest WSS appeared to have notable AWE, particularly in the cases of the $\mathrm{P}_{4}$ AComA, $\mathrm{P}_{5} \mathrm{MCA}$, and $\mathrm{P}_{5} \mathrm{AComA}$.

\section{Univariate Analysis of Differences Between the AWE and No-AWE Groups}

Results of the univariate analysis for morphological and hemodynamic factors are summarized in Table 2. For hemodynamic factors, the AWE group had significantly lower WSS (3.88 vs $11.69 \mathrm{~Pa}, \mathrm{p}=0.00287)$, WSS* $(0.33$ vs $0.90, \mathrm{p}<0.001)$, and average velocity $(0.13$ vs $0.27 \mathrm{~m} /$ sec, $\mathrm{p}=0.00287)$, while OSI (0.04 vs 0.03, $\mathrm{p}=0.0694)$ and SPI (0.10 vs $0.12, \mathrm{p}=0.613)$ showed no statistically 

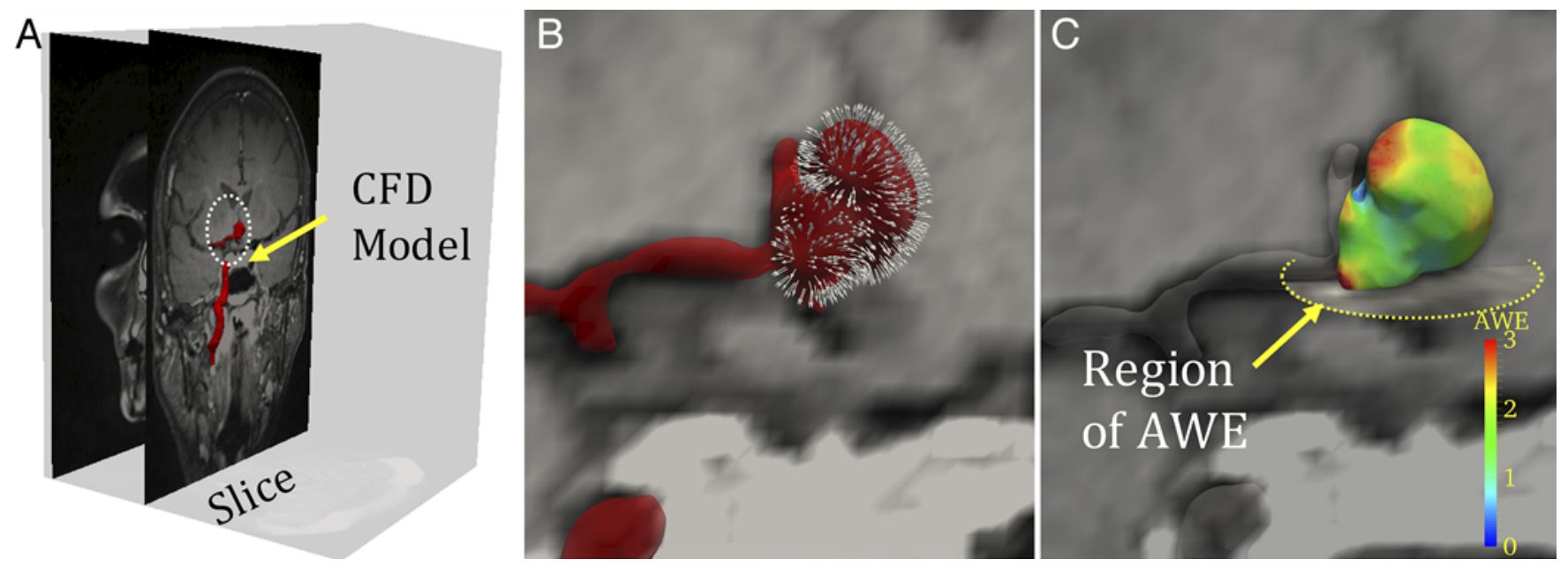

FIG. 1. Pipeline to register the CFD model to postcontrast VWMRI and quantify AWE. A: CFD model registered to the VWMRI using landmark-based image registration. B: Vectors pointing normal to the aneurysm surface, which were used to compute MRI-SI at each point on the surface. The MRI-SI was then normalized to the nominal intensity of the image volume. C: Normalized MRI-SI maps where the maximum intensity corresponds to the region that showed AWE. Figure is available in color online only.

significant differences between the 2 groups. Among morphological factors, the AWE group IAs had larger dome size ( 7.22 vs $3.61 \mathrm{~mm}, \mathrm{p}=0.00462)$ and SR (4.26 vs 2.33 , $\mathrm{p}=0.0251)$. Other morphological factors, including AR (1.65 vs $1.48, \mathrm{p}=0.613)$, BNF (1.48 vs $1.43, \mathrm{p}=0.926)$, HWR (1.10 vs $0.92, \mathrm{p}=0.0698)$, and NSI (0.13 vs 0.06 , $\mathrm{p}$ $=0.0695)$, showed no significant differences between the AWE and no-AWE groups.

\section{Multivariate Logistic Regression on Significant Variables}

Figure $3 \mathrm{~A}$ and $\mathrm{B}$ shows a univariate logistic predic-

TABLE 1. Demographic information of the studied cohort

\begin{tabular}{lccc}
\hline Parameter & AWE & No AWE & p Value \\
\hline Mean age (yrs) & 50.8 & 56.7 & 0.29 \\
\hline Hypertension & $50 \%$ & $63 \%$ & 0.99 \\
\hline Sex & & & 1 \\
\hline Female & 4 & 8 & \\
\hline Male & 4 & 3 & \\
\hline IA location & & & 0.12 \\
\hline MCA & 3 & 11 & 0.4 \\
\hline AComA & 5 & 5 & 0.36 \\
\hline PComA & 1 & 0 & 0.12 \\
\hline Total & 9 & 16 & 0.69 \\
\hline Smoking & $33 \%$ & $44 \%$ & 0.88 \\
\hline Hyperlipidemia & $11 \%$ & $13 \%$ & NA \\
\hline Diabetes & $0 \%$ & $0 \%$ & \\
\hline IA side & & & \\
\hline Left & 1 & 4 & \\
\hline Right & 3 & 6 & \\
\hline Both & 0 & 1 & \\
\hline
\end{tabular}

NA $=$ not applicable.

Values are presented as number or percentage of patients unless otherwise indicated. tive model, using only the square root of the WSS as the predictor for the AWE, which was found to be a significant predictor $(\mathrm{p}=0.01, \mathrm{AIC}=22.7)$ along with $\mathrm{WSS}^{*}$. As shown in Fig. 3C, introducing the $\log ($ size) reduced the AIC to 17.6, at the cost of making the square root of WSS insignificant and showing only a trend for $\log$ (size) $(\mathrm{p}=0.078)$. For comparison, Fig. 3D shows a predictive model relying on raw, nontransformed variables, which was found to be similar to the nonlinear model.

The performance of both models was assessed for their accuracy and AUC. As shown in Fig. 3E, the univariate model reached an accuracy of 0.84 , with an AUC of 0.9 on the training data. On the other hand, the bivariate model, shown in Fig. 3F, with $\log$ (size) as the second predictor, had an accuracy of 0.92 and an AUC of 0.95. We internally validated and calibrated both models using bootstrapping with 1000 resamples. The accuracy was 0.78 for the univariate model and 0.84 for the bivariate one. For both models, a Hosmer-Lemeshow test showed no significant deviations between the model predictions and grouped relative frequencies $(p=0.62$ and $p=0.98$ for the univariate and bivariate models, respectively). McFadden's $\mathrm{R}^{2}$ values were 0.43 and 0.64 , respectively.

\section{Correlation Between MRI-SIF and WSS in the AWE Group}

There was a qualitative correlation between regions of maximum MRI-SIF and regions of low WSS* (Fig. 4). In particular, we observed that 2 cases in which IAs showed distinct and focal enhancement in Fig. 1, namely $\mathrm{P}_{5}$ AComA and $\mathrm{P}_{2}$ AComA, were those with IA blebs. From Fig. 4 we noted that the focal enhancement was located within the bleb region, and interestingly, also coincided with the location of lowest WSS* on the aneurysm dome.

As shown in Fig. 5A and B, we observed a weak correlation of MRI-SIF with WSS $\left(r^{2}=0.23, p=0.019\right)$ and a moderate correlation with WSS* $(r=0.47, p=0.041)$. To further explore this association, we computed MRI-SIF in regions of low, intermediate, and high WSS*. The means \pm 

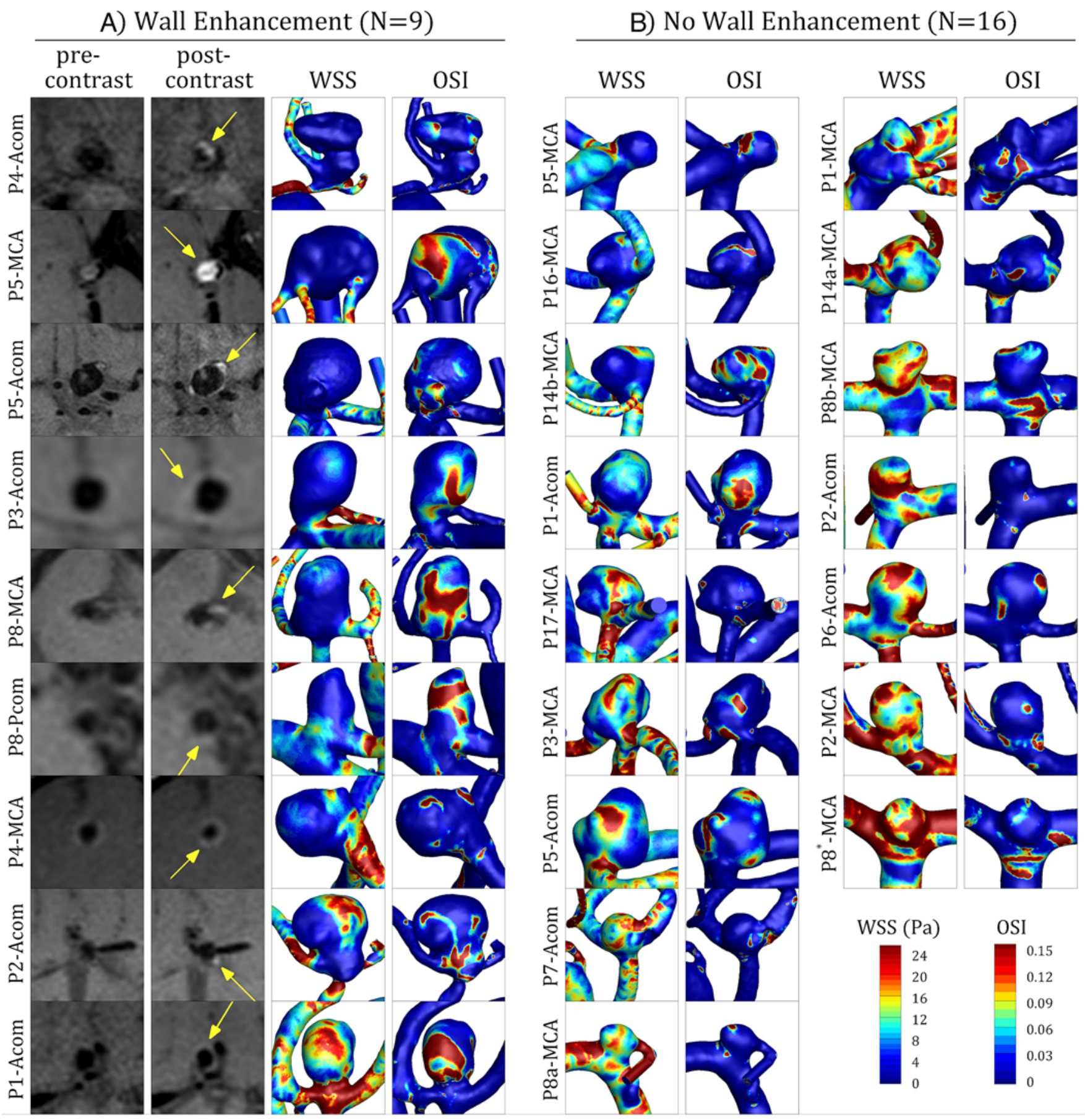

FIG. 2. Hemodynamic maps of aneurysms with and without AWE, arranged in ascending order from lowest to highest WSS. A: The first 2 columns show pre- and postcontrast images of aneurysms with AWE, where the region of enhancement is marked with an arrow. The next 2 columns display WSS and OSI maps, respectively. B: WSS and OSI maps for aneurysms without AWE. For brevity, pre- and postcontrast images are not displayed. Note that case names identify the aneurysm location: anterior communicating artery (Acom), MCA, or posterior communicating artery (Pcom). Figure is available in color online only.

standard deviations of low-, intermediate-, and high-WSS* regions were $0.063 \pm 0.027,0.18 \pm 0.020$, and $0.56 \pm 0.16$, and the corresponding MRI-SIF values in these regions were $1.41 \pm 0.61,1.17 \pm 0.28$, and $1.15 \pm 0.31$, respectively. As shown in Fig. 5C, MRI-SIF values in the low-WSS* re- gions were statistically different from those in the regions of intermediate $(p=0.018)$ and high $(p<0.001) \mathrm{WSS}^{*}$, but there were no statistically significant differences between the MRI-SIF values in the intermediate- and high-WSS* regions $(\mathrm{p}=0.085)$. 
TABLE 2. Results from univariate statistical analysis for all parameters examined in the AWE and no-AWE groups

\begin{tabular}{lccl}
\hline \multicolumn{1}{c}{ Parameter } & AWE $(n=9)$ & No AWE $(n=16)$ & $p$ Value \\
\hline Hemodynamics & & & \\
\hline WSS $(\mathrm{Pa})$ & $3.88 \pm 3.09$ & $11.69 \pm 6.15$ & $\mathbf{0 . 0 0 2 8 7}$ \\
\hline WSS $^{*}$ & $0.33 \pm 0.20$ & $0.90 \pm 0.43$ & $\mathbf{0 . 0 0 0 0 7 1}$ \\
\hline OSI & $0.04 \pm 0.03$ & $0.03 \pm 0.02$ & 0.0694 \\
\hline SPI & $0.10 \pm 0.10$ & $0.12 \pm 0.08$ & 0.613 \\
\hline Mean velocity (m/sec) & $0.13 \pm 0.07$ & $0.27 \pm 0.09$ & $\mathbf{0 . 0 0 2 8 7}$ \\
\hline IA morphology & & & \\
\hline Size $(\mathrm{mm})$ & $7.22 \pm 3.63$ & $3.61 \pm 1.24$ & $\mathbf{0 . 0 0 4 6 2}$ \\
\hline AR & $1.65 \pm 0.68$ & $1.48 \pm 0.49$ & 0.613 \\
\hline SR & $4.26 \pm 2.42$ & $2.33 \pm 0.81$ & $\mathbf{0 . 0 2 5 1}$ \\
\hline BNF & $1.48 \pm 0.73$ & $1.43 \pm 0.46$ & 0.926 \\
\hline HW & $1.10 \pm 0.23$ & $0.92 \pm 0.17$ & 0.0698 \\
\hline NSI & $0.13 \pm 0.08$ & $0.06 \pm 0.03$ & 0.0695 \\
\hline
\end{tabular}

Values are presented as mean \pm SD unless otherwise indicated. Boldface type indicates statistical significance.

\section{Discussion}

We have used VWMRI to investigate the associations between AWE of unruptured IAs and hemodynamic and morphological factors typically associated with ruptured IAs. The presence of AWE was associated with morphological risk factors, particularly size and SR, and hemodynamic factors such as WSS, but only WSS was found to be an independent predictor of AWE.

\section{Relationship Between AWE and IA Wall Inflammation}

Wall inflammation is thought to be a key component in IA wall degradation and rupture. Recently, Larsen et al. performed histological analysis in 13 patients who underwent VWMRI before their IA clipping surgery. ${ }^{24}$ Five patients showed strong AWE, and histological analysis revealed inflammatory cell infiltrations in 4 of these patients through myeloperoxidase staining and neovascularization in 1 patient. On the other hand, remaining aneurysms without AWE showed no signs of inflammatory cell invasion or neovascularization; thus, AWE occurred only when histological signs of inflammation were present. Cebral et al. demonstrated the association between hemodynamics and histology of tissues resected during
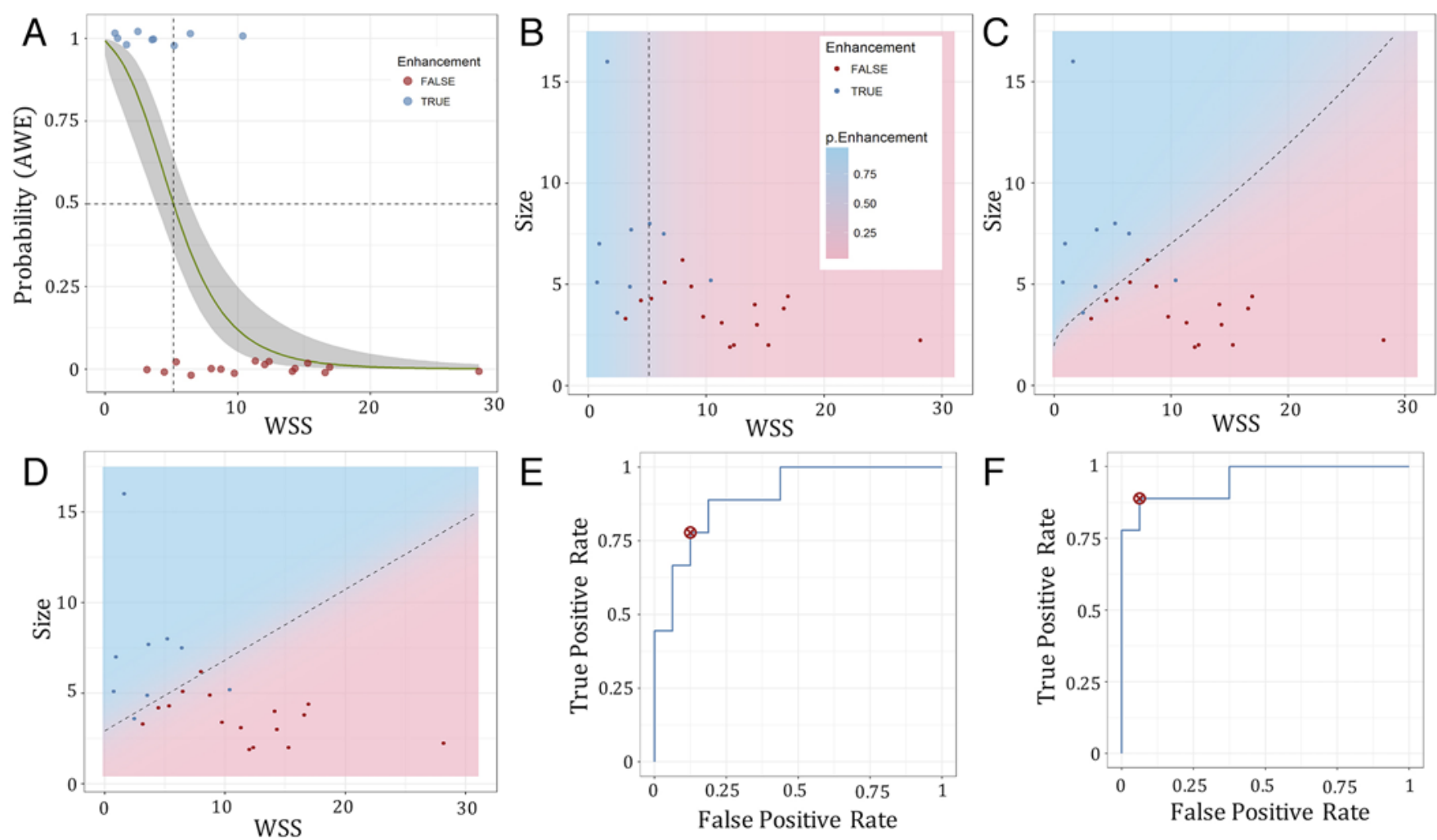

FIG. 3. A: A univariate logistic predictive model using only the square root of WSS as the predictor for AWE. Noise is added to the $y$-axis for better visualization. For all subplots, blue and red dots refer to the AWE and no-AWE groups, respectively. The green line is the logistic regression curve, and the gray band is the $95 \% \mathrm{Cl}$. B: The same univariate model shown in panel A visualized as an area. The dashed line indicates $50 \%$ probability of AWE. C: A bivariate model, using the square root of WSS and log(size) as the predictors of AWE. Due to nonlinear transformation of variables, the $50 \%$ probability boundary (dashed line) is not a straight line. D: An alternative bivariate model, using raw WSS and size values as predictors of AWE. This model is only marginally less accurate than the nonlinear model shown in panel C. E: ROC curve for the univariate predictors. F: ROC curve for the bivariate predictors. The red mark in panels $\mathrm{E}$ and $\mathrm{F}$ denotes the point where the highest accuracy is achieved. Figure is available in color online only. 

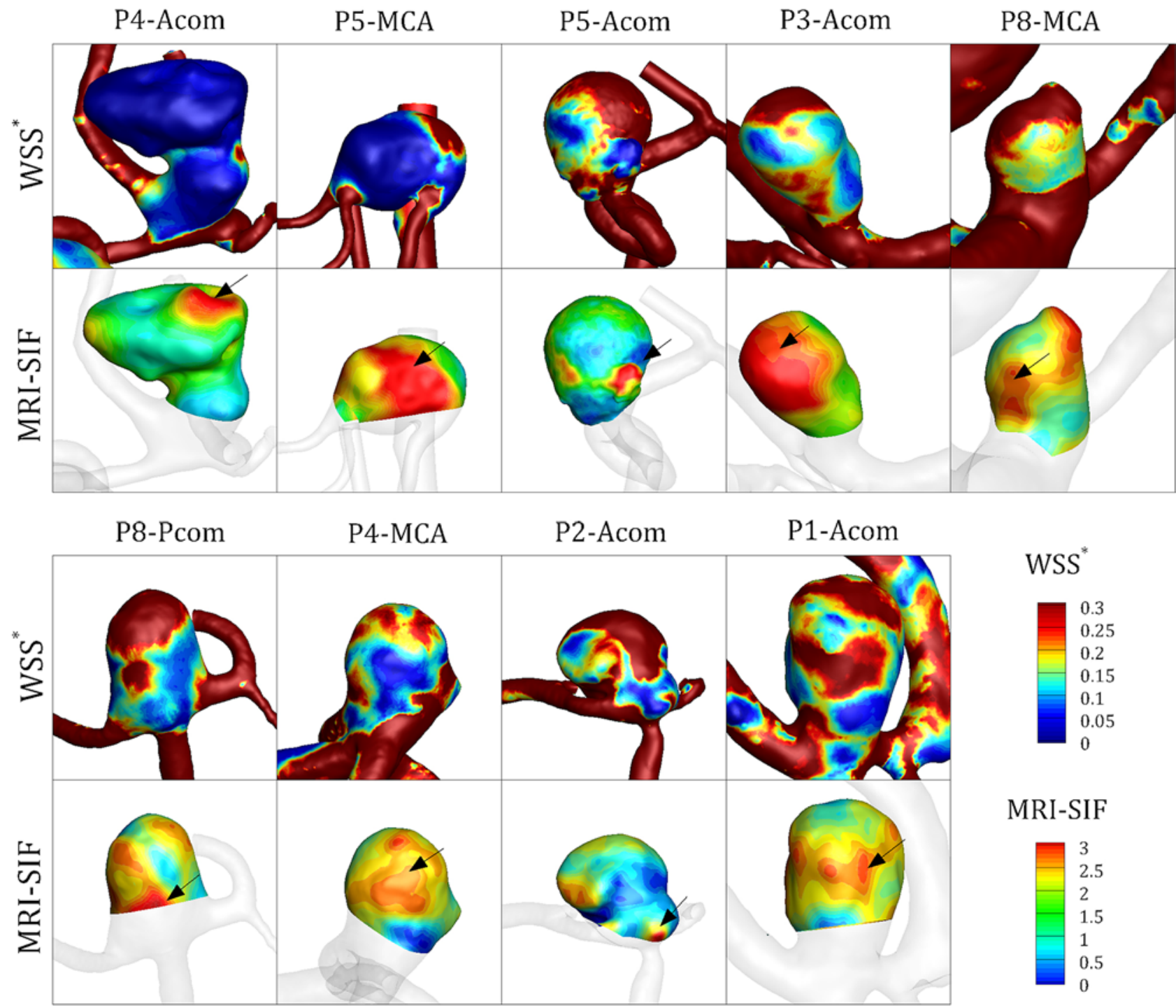

FIG. 4. WSS* and MRI-SIF maps of aneurysms that showed AWE. The arrows in the MRI-SIF maps point to the regions that were independently evaluated by 2 neuroradiologists as the regions of postcontrast AWE. Figure is available in color online only.

IA surgery for 9 ruptured and 11 unruptured IAs. ${ }^{4}$ Those authors found that high WSS was correlated with regions of wall inflammation and low flow was correlated with degenerated wall that had lost mural cells, both of which are markers of unstable IAs.

Since the AWE group in our study had low flow and WSS, a mural cell-mediated inflammatory response may have been at play. Previous studies have shown that aneurysms that are missing mural smooth muscle cells are incapable of organizing a luminal thrombus, a condition that may subsequently lead to an increased inflammatory reaction and thus severe wall degradation and aneurysm growth and rupture..$^{28}$ On the other hand, it is also plausible that AWE may not be a direct marker of structural changes and wall inflammation, but rather could be an epiphenomenon related to growth or other remodeling processes that may predispose IAs to rupture.

\section{Relationship Between Hemodynamics and Aneurysm Remodeling}

The majority of the evidence linking WSS to IA rupture is derived from retrospective CFD studies that have compared hemodynamics in unruptured versus already ruptured IAs. In particular, 2 large, retrospective CFD studies independently found low $\mathrm{WSS}^{39}(\mathrm{n}=119$ patients) and high $\operatorname{WSS}^{6}(\mathrm{n}=210$ patients) to be associated with rupture status. While there have been other studies of the associations of WSS with rupture, these 2 seminal studies highlight an ongoing controversy in aneurysm literature: is high or low WSS more deleterious? A unifying hypothesis has been proposed whereby both low and high WSS may drive distinct mechanobiological pathways toward aneurysm instability. ${ }^{31}$

Investigating this hypothesis, Cebral et al. recently 

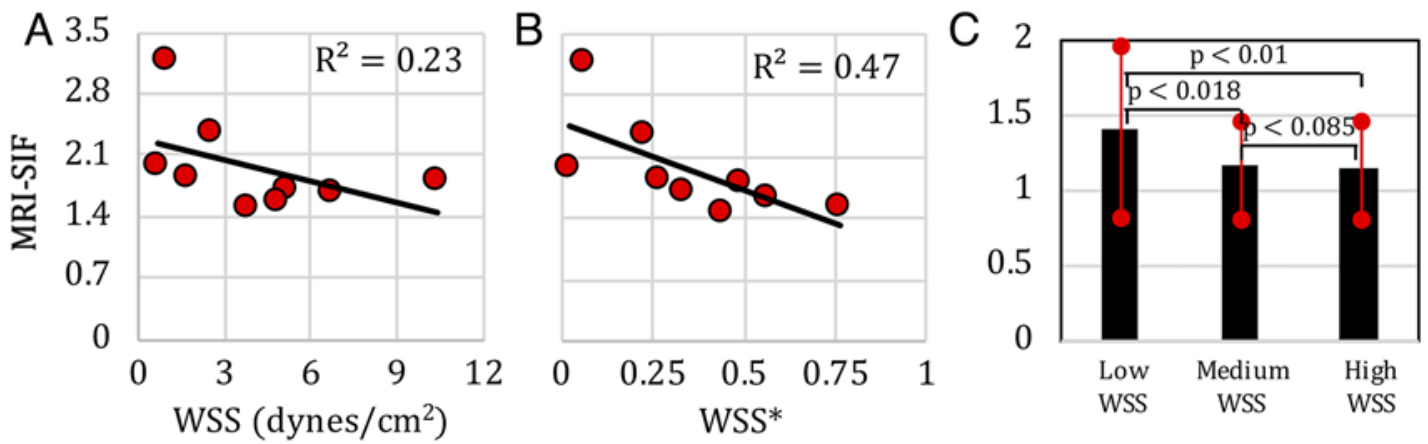

FIG. 5. Quantitative correlation of the sac-averaged MRI-SIF with (A) WSS and (B) WSS*. C: MRI-SIF in regions of low, intermediate, and high WSS*. The red line indicates the standard deviation. Figure is available in color online only.

demonstrated that regions visually characteristic of atherosclerotic and hyperplastic walls tended to have focally low flow and WSS while thin-walled regions had high WSS. ${ }^{5}$ In our study, we found AWE to occur at the location of focally low WSS, as shown in Fig. 2. In particular, the 2 IAs that showed distinct and focal enhancement $\left(\mathrm{P}_{5} \mathrm{AComA}\right.$ and $\mathrm{P}_{2} \mathrm{AComA}$ ) coincided with the location of the lowest WSS on the IA sac, and interestingly, were also aneurysms that had blebs. Since bleb regions tend to have slow and recirculating flow, ${ }^{17}$ it is plausible that these 2 IAs had atherosclerotic and hyperplastic walls as suggested by the findings of Cebral et al. ${ }^{5}$ Although previous studies have indicated that IA walls that appear atherosclerotic and hyperplastic are thicker, this characteristic does not necessarily imply that such regions are mechanistically stronger and not rupture prone. As suggested by Cebral et al., ${ }^{5}$ it is plausible that the wall structure in these low-WSS regions may be degraded, resulting in an overall weaker IA wall.

\section{Aneurysm Wall Enhancement in Rupture Risk Assessment}

Recently VWMRI has been used in patients with IAs, and AWE on VWMRI is considered to be a marker of wall inflammation, and thus aneurysm wall weakening. Matouk et al. showed the presence of AWE in 5 of 5 ruptured aneurysms. ${ }^{30}$ Similarly, Nagahata et al. investigated 61 ruptured and 83 unruptured IAs and found strong to faint signs of AWE in $98.4 \%$ of ruptured and only $18.1 \%$ of unruptured IAs. ${ }^{33}$ Distinguishing strong from faint enhancement is a subjective process, and thus Wang et al. quantitatively measured enhancement ratios of IA walls, bodies, and necks on pre- versus postcontrast images, with a threshold value of $61.5 \%$ to distinguish ruptured from unruptured IAs. ${ }^{36}$ However, issues such as leaked or stagnant contrast agent and alteration of radiological characteristics postrupture may limit the applicability of these studies to rupture risk assessment.

Edjlali et al. focused instead on unruptured IAs and prospectively found AWE to be present in $87 \%$ of 31 unstable and symptomatic IAs and only $28.5 \%$ of 77 stable aneurysms..$^{11}$ Of 108 patients, 46\% showed AWE, which is comparable to $36 \%$ of the 25 IAs showing AWE in the present study. More recently, Lv et al. also focused on unruptured IAs and found the presence of AWE to be associated with conventional rupture-related risk factors such as larger size and irregular shape, findings consistent with those of the present study. ${ }^{27}$ These findings suggest that AWE may be a potential patient-specific biomarker that may be complementary to the conventional risk factors for IA rupture.

\section{Association Between Morphology and Rupture Risk Assessment}

The most widely used rupture risk assessment method in clinical practice is the aneurysm size measured from DSA, which was established by the International Study of Unruptured Intracranial Aneurysms (ISUIA). ${ }^{38}$ However, one major drawback of the size-based ISUIA standard is that small-sized aneurysms may still rupture. Juvela et al. showed that $37 \%$ of patients had IAs smaller than $5 \mathrm{~mm}$ in diameter ${ }^{15}$ which would be within ISUIA grade 1 (lowest risk score), defined as IA size $<7 \mathrm{~mm}$. In fact, Korja et al. found that during lifelong follow-up of 118 patients with unruptured IAs, $19 \%$ were $<7 \mathrm{~mm}$ at the time of rupture. ${ }^{23}$ Similar findings in ruptured mirror aneurysms were reported by Maslehaty et al., where the average size of ruptured aneurysms was $7 \mathrm{~mm} .{ }^{29}$ In our study, although the average IA size of the AWE group was $7.22 \mathrm{~mm}, 4$ of 9 IAs that showed AWE were smaller than $7 \mathrm{~mm}$, falling under the ISUIA low-risk category despite showing AWE.

A significant correlation between IA size and the presence of AWE has been reported by several authors. In particular, Liu et al. found aneurysm size to be an independent predictor of AWE. ${ }^{25}$ These authors found $83 \%$ of 36 IAs $>7 \mathrm{~mm}$ to have AWE, while only $12 \%$ of $25 \mathrm{IAs}<7 \mathrm{~mm}$ showed AWE. We similarly found all IAs $>7 \mathrm{~mm}$ to have AWE, and only $25 \%$ of IAs $<7 \mathrm{~mm}$ to have AWE. This higher prevalence of smaller IAs in our AWE group may be due to the small sample size; however, these findings suggest that AWE may provide additional information about aneurysm instability other than size, and thus could be a potential metric to improve the current standards for assessment of rupture risk.

Shape-based metrics have been shown to be better predictors of rupture risk than size alone. Interestingly, we did not find AR to be a statistically significant predictor of AWE. Previous studies have shown AR to be a potential metric to assess rupture risk; ${ }^{37}$ however, the findings have not been unequivocal ${ }^{39}$ and are based on retrospective data. On the other hand, a recent longitudinal study of 93 patients showed that in growing versus stable aneurysms, 
AR did not show any significance, even when aneurysms were segregated by size $(<3,3-5,5-7$, or $>7 \mathrm{~mm})$ or location. ${ }^{8}$ The only 2 parameters that enabled consistent detection of growing versus stable aneurysms were NSI and SR, both of which showed significance or a trend toward significance in detecting aneurysms with wall enhancement in our study.

Location of IAs has previously been suggested as a risk factor for rupture. The ISUIA study results suggested that aneurysms located at the posterior circulation have the highest risk of rupture, while both ICA and MCA aneurysms have low to moderate risks of rupture. ${ }^{38}$ Although we did not evaluate location-specific quantities, whether hemodynamics vary based on location is not clear. In particular, Chien et al. showed higher WSS in MCA than the posterior communicating artery (PComA), ${ }^{7}$ while Varble et al. found no hemodynamic differences in MCA and PComA aneurysms. ${ }^{35}$

\section{Clinical Implications}

With the increasing number of incidental findings of unruptured IAs, patient-specific assessment of rupture risk is gaining importance. Recent efforts have been focused on VWMRI; ${ }^{11,30}$ however, this imaging technique is not available in standard clinical care or may not be feasible due to contraindications. On the other hand, CFD-derived hemodynamic factors, such as WSS and OSI, have shown promise for aneurysm rupture risk assessment. Our study has shown that low WSS is an independent predictor of AWE and thus may serve as an alternative measure to identify high-risk aneurysms in need of immediate treatment.

We have also provided quantitative evidence that AWE on VWMRI can be augmented with CFD-based hemodynamic factors to investigate the degraded state of the vessel wall. While we did not perform a validation of AWE against histological data, there is sufficient evidence in the literature to suggest that AWE is a marker of IA wall degeneration, ${ }^{11,24,30}$ and thus CFD-based hemodynamic factors may provide a quantitative measure to study aneurysmal wall remodeling in vivo by longitudinally following patients who have not been treated.

Although larger aneurysm size is highly correlated with rupture, $25 \%$ of small aneurysms also rupture. Toward developing improved predictive markers for small aneurysms, parsimonious models that incorporate our proposed parameters, MRI-SIF with WSS, may allow better assessment of the rupture risk of small aneurysms.

\section{Limitations}

One key limitation of the current study is the small sample size. While we have found a correlation between low WSS and the presence of AWE, a larger sample size with radiological follow-up is needed to establish the usefulness of hemodynamics as a biomarker of AWE and rupture risk. Although previous studies have already found AWE to be present in IAs with wall inflammation, the histological data of patients with AWE were not available in our study, and thus direct association of wall pathology with hemodynamic factors could not be investigated. Lastly, we have made conventional assumptions in our CFD model such as rigid walls, Newtonian rheology, and fully developed inflow. These are commonly accepted assumptions and their effects are now well documented to be of relatively minor importance.

\section{Conclusions}

Our results suggest that the presence of AWE in our unruptured IA cohort is associated with conventional hemodynamic and morphological factors. Aneurysms with larger size and SR were more prone to have AWE. However, only low WSS was independently predictive of AWE. Our results support the hypothesis that low WSS in the AWE group may indicate a growth and remodeling process that may predispose such aneurysms to rupture; however, a causality between the two could not be established. VWMRI may be augmented with CFD-based hemodynamic factors to assess patient-specific risk factors.

\section{Acknowledgments}

Computations were performed on the GPC supercomputer at the SciNet HPC Consortium. ${ }^{26}$ SciNet is funded by the Canada Foundation for Innovation under the auspices of Compute Canada, the Government of Ontario, the Ontario Research FundResearch Excellence, and the University of Toronto. M.O.K. acknowledges postdoctoral fellowship funding from the Natural Sciences and Engineering Research Council of Canada.

\section{References}

1. Bazilevs Y, Hsu M, Zhang Y, Wang W, Liang X, Kvamsdal T, et al: A fully-coupled fluid-structure interaction simulation of cerebral aneurysms. Comput Mech 46:3-16, 2010

2. Berg P, Roloff C, Beuing O, Voss S, Sugiyama S, Aristokleous N, et al: The Computational Fluid Dynamics Rupture Challenge 2013-Phase II: variability of hemodynamic simulations in two intracranial aneurysms. J Biomech Eng 137:121008, 2015

3. Berg P, Voß S, Saalfeld S, Janiga G, Bergersen AW, ValenSendstad K, et al: Multiple Aneurysms AnaTomy CHallenge 2018 (MATCH): Phase I: segmentation. Cardiovasc Eng Technol 9:565-581, 2018

4. Cebral J, Ollikainen E, Chung BJ, Mut F, Sippola V, Jahromi $\mathrm{BR}$, et al: Flow conditions in the intracranial aneurysm lumen are associated with inflammation and degenerative changes of the aneurysm wall. AJNR Am J Neuroradiol 38:119-126, 2017

5. Cebral JR, Detmer F, Chung BJ, Choque-Velasquez J, Rezai $\mathrm{B}$, Lehto $\mathrm{H}$, et al: Local hemodynamic conditions associated with focal changes in the intracranial aneurysm wall. AJNR Am J Neuroradiol 40:510-516, 2019

6. Cebral JR, Mut F, Weir J, Putman C: Quantitative characterization of the hemodynamic environment in ruptured and unruptured brain aneurysms. AJNR Am J Neuroradiol 32:145-151, 2011

7. Chien A, Tateshima S, Castro M, Sayre J, Cebral J, Viñuela F: Patient-specific flow analysis of brain aneurysms at a single location: comparison of hemodynamic characteristics in small aneurysms. Med Biol Eng Comput 46:1113-1120, 2008

8. Chien A, Xu M, Yokota H, Scalzo F, Morimoto E, Salamon $\mathrm{N}$ : Nonsphericity index and size ratio identify morphologic differences between growing and stable aneurysms in a longitudinal study of 93 cases. AJNR Am J Neuroradiol 39:500-506, 2018

9. Dempere-Marco L, Oubel E, Castro M, Putman C, Frangi A, 
Cebral J: CFD analysis incorporating the influence of wall motion: application to intracranial aneurysms. In Larsen R, Nielsen M, Sporring J (eds): Medical Image Computing and Computer-Assisted Intervention-MICCAI 2006. Lecture Notes in Computer Science, Vol 4191. Berlin: Springer, 2006, pp 438-445

10. Dhar S, Tremmel M, Mocco J, Kim M, Yamamoto J, Siddiqui $\mathrm{AH}$, et al: Morphology parameters for intracranial aneurysm rupture risk assessment. Neurosurgery 63:185-197, 2008

11. Edjlali M, Gentric JC, Régent-Rodriguez C, Trystram D, Hassen WB, Lion S, et al: Does aneurysmal wall enhancement on vessel wall MRI help to distinguish stable from unstable intracranial aneurysms? Stroke 45:3704-3706, 2014

12. Fung C, Mavrakis E, Filis A, Fischer I, Suresh M, Tortora A, et al: Anatomical evaluation of intracranial aneurysm rupture risk in patients with multiple aneurysms. Neurosurg Rev 42:539-547, 2019

13. Geers AJ, Larrabide I, Radaelli AG, Bogunovic H, Kim M, Gratama van Andel HA, et al: Patient-specific computational hemodynamics of intracranial aneurysms from 3D rotational angiography and CT angiography: an in vivo reproducibility study. AJNR Am J Neuroradiol 32:581-586, 2011

14. Hoi Y, Wasserman BA, Xie YJ, Najjar SS, Ferruci L, Lakatta EG, et al: Characterization of volumetric flow rate waveforms at the carotid bifurcations of older adults. Physiol Meas 31:291-302, 2010

15. Juvela S, Porras M, Poussa K: Natural history of unruptured intracranial aneurysms: probability of and risk factors for aneurysm rupture. J Neurosurg 93:379-387, 2000

16. Kallmes DF: Identifying "truth" in computational fluid dynamics research. AJNR Am J Neuroradiol 32:E122, E123, 2011

17. Kawaguchi T, Nishimura S, Kanamori M, Takazawa H, Omodaka S, Sato K, et al: Distinctive flow pattern of wall shear stress and oscillatory shear index: similarity and dissimilarity in ruptured and unruptured cerebral aneurysm blebs. J Neurosurg 117:774-780, 2012

18. Khan M, Valen-Sendstad K, Steinman D: Direct numerical simulation of laminar-turbulent transition in a non-axisymmetric stenosis model for Newtonian vs. shear-thinning nonNewtonian rheologies. Flow Turbul Combust 102:43-72, 2019

19. Khan MO, Chnafa C, Gallo D, Molinari F, Morbiducci U, Steinman DA, et al: On the quantification and visualization of transient periodic instabilities in pulsatile flows. J Biomech 52:179-182, 2017

20. Khan MO, Steinman DA, Valen-Sendstad K: Non-Newtonian versus numerical rheology: practical impact of shear-thinning on the prediction of stable and unstable flows in intracranial aneurysms. Int J Numer Methods Biomed Eng 33:e2836, 2017

21. Khan MO, Valen-Sendstad K, Steinman DA: Cerebral aneurysm blood flow simulations: there's solver settings and then there's solver settings. J Biomech 61:280, 2017 (Letter)

22. Khan MO, Valen-Sendstad K, Steinman DA: Narrowing the expertise gap for predicting intracranial aneurysm hemodynamics: impact of solver numerics versus mesh and time-step resolution. AJNR Am J Neuroradiol 36:1310-1316, 2015

23. Korja M, Lehto H, Juvela S: Lifelong rupture risk of intracranial aneurysms depends on risk factors: a prospective Finnish cohort study. Stroke 45:1958-1963, 2014

24. Larsen N, von der Brelie C, Trick D, Riedel C, Linder T, Madjidyar J, et al: Vessel wall enhancement in unruptured aneurysms: an indicator for higher risk of rupture? Highresolution MR imaging and correlated histologic findings. AJNR Am J Neuroradiol 39:1617-1621, 2018

25. Liu P, Qi H, Liu A, Lv X, Jiang Y, Zhao X, et al: Relationship between aneurysm wall enhancement and conventional risk factors in patients with unruptured intracranial aneurysms: a black-blood MRI study. Interv Neuroradiol 22:501-505, 2016

26. Loken C, Gruner D, Groer L, Peltier R, Bunn N, Craig M, et al: SciNet: lessons learned from building a power-efficient top-20 system and data centre. J Phys Conf Ser 256:012026, 2010

27. Lv N, Karmonik C, Chen S, Wang X, Fang Y, Huang Q, et al: Relationship between aneurysm wall enhancement in vessel wall magnetic resonance imaging and rupture risk of unruptured intracranial aneurysms. Neurosurgery 84:E385-E391, 2019

28. Marbacher S, Marjamaa J, Bradacova K, von Gunten M, Honkanen P, Abo-Ramadan U, et al: Loss of mural cells leads to wall degeneration, aneurysm growth, and eventual rupture in a rat aneurysm model. Stroke 45:248-254, 2014

29. Maslehaty H, Capone C, Frantsev R, Fischer I, Jabbarli R, Cornelius JF, et al: Predictive anatomical factors for rupture in middle cerebral artery mirror bifurcation aneurysms. J Neurosurg 128:1799-1807, 2018

30. Matouk CC, Mandell DM, Günel M, Bulsara KR, Malhotra A, Hebert R, et al: Vessel wall magnetic resonance imaging identifies the site of rupture in patients with multiple intracranial aneurysms: proof of principle. Neurosurgery 72:492-496, 2013

31. Meng H, Tutino VM, Xiang J, Siddiqui A: High WSS or low WSS? Complex interactions of hemodynamics with intracranial aneurysm initiation, growth, and rupture: toward a unifying hypothesis. AJNR Am J Neuroradiol 35:1254-1262, 2014

32. Mortensen M, Valen-Sendstad K: Oasis: a high-level/highperformance open source Navier-Stokes. Comput Phys Commun 188:177-188, 2015

33. Nagahata S, Nagahata M, Obara M, Kondo R, Minagawa N, Sato S, et al: Wall enhancement of the intracranial aneurysms revealed by magnetic resonance vessel wall imaging using three-dimensional turbo spin-echo sequence with motionsensitized driven-equilibrium: a sign of ruptured aneurysm? Clin Neuroradiol 26:277-283, 2016

34. Valen-Sendstad K, Bergersen AW, Shimogonya Y, Goubergrits L, Bruening J, Pallares J, et al: Real-world variability in the prediction of intracranial aneurysm wall shear stress: the 2015 International Aneurysm CFD Challenge. Cardiovasc Eng Technol 9:544-564, 2018

35. Varble N, Rajabzadeh-Oghaz H, Wang J, Siddiqui A, Meng $\mathrm{H}$, Mowla A: Differences in morphologic and hemodynamic characteristics for "PHASES-based" intracranial aneurysm locations. AJNR Am J Neuroradiol 38:2105-2110, 2017

36. Wang GX, Wen L, Lei S, Ran Q, Yin JB, Gong ZL, et al: Wall enhancement ratio and partial wall enhancement on MRI associated with the rupture of intracranial aneurysms. J Neurointerv Surg 10:566-570, 2018

37. Weir B, Amidei C, Kongable G, Findlay JM, Kassell NF, Kelly J, et al: The aspect ratio (dome/neck) of ruptured and unruptured aneurysms. J Neurosurg 99:447-451, 2003

38. Wiebers DO, Whisnant JP, Huston J III, Meissner I, Brown RD Jr, Piepgras DG, et al: Unruptured intracranial aneurysms: natural history, clinical outcome, and risks of surgical and endovascular treatment. Lancet 362:103-110, 2003

39. Xiang J, Natarajan SK, Tremmel M, Ma D, Mocco J, Hopkins LN, et al: Hemodynamic-morphologic discriminants for intracranial aneurysm rupture. Stroke 42:144-152, 2011

40. Xiao W, Qi T, He S, Li Z, Ou S, Zhang G, et al: Low wall shear stress is associated with local aneurysm wall enhancement on high-resolution MR vessel wall imaging. AJNR Am J Neuroradiol 39:2082-2087, 2018

41. Zarrinkoob L, Ambarki K, Wåhlin A, Birgander R, Eklund A, Malm J: Blood flow distribution in cerebral arteries. J Cereb Blood Flow Metab 35:648-654, 2015 


\section{Disclosures}

The authors report no conflict of interest concerning the materials or methods used in this study or the findings specified in this paper.

\section{Author Contributions}

Conception and design: all authors. Acquisition of data: all authors. Analysis and interpretation of data: all authors. Drafting the article: all authors. Critically revising the article: all authors.
Reviewed submitted version of manuscript: all authors. Approved the final version of the manuscript on behalf of all authors: Khan. Statistical analysis: all authors. Administrative/technical/material support: all authors. Study supervision: all authors.

\section{Correspondence}

Muhammad Owais Khan: Stanford University, Stanford, CA. mokhan@stanford.edu. 